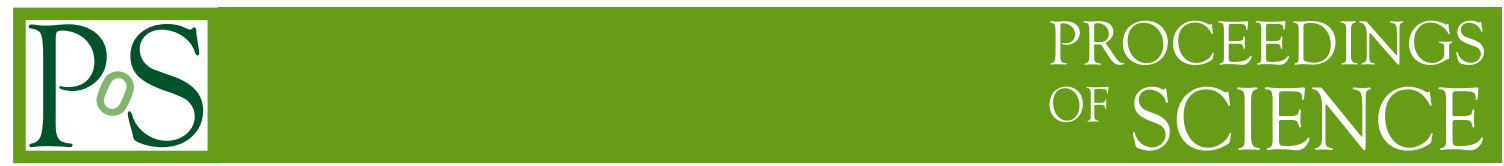

\title{
Indirect detection of GRBs by ionospheric response
}

\section{Rudolf Slošiar}

Astronomical Union of Amateur Astronomers, Bojnice, Slovak Republic

E-mail: rstrudyepost.sk

\section{René Hudec}

Astronomical Institute of the Academy of Sciences of the Czech Republic

E-mail: rene.hudec@gmail.com

We report on the independent and indirect detection of GRBs by their ionospheric response (SIDSudden Ionospheric Disturbance) observed at VLF (Very Low Frequency). Although few such detections have been already reported in the past, the capability of such alternative and indirect investigations of GRBs still remains to be investigated in more details. We present and discuss examples of such VLF/SID detections in three cases (GRB 060124A, GRB080319D and GRB080320A).

VII Microquasar Workshop: Microquasars and Beyond September 1 - 5, 2008

Foca, Izmir, Turkey 

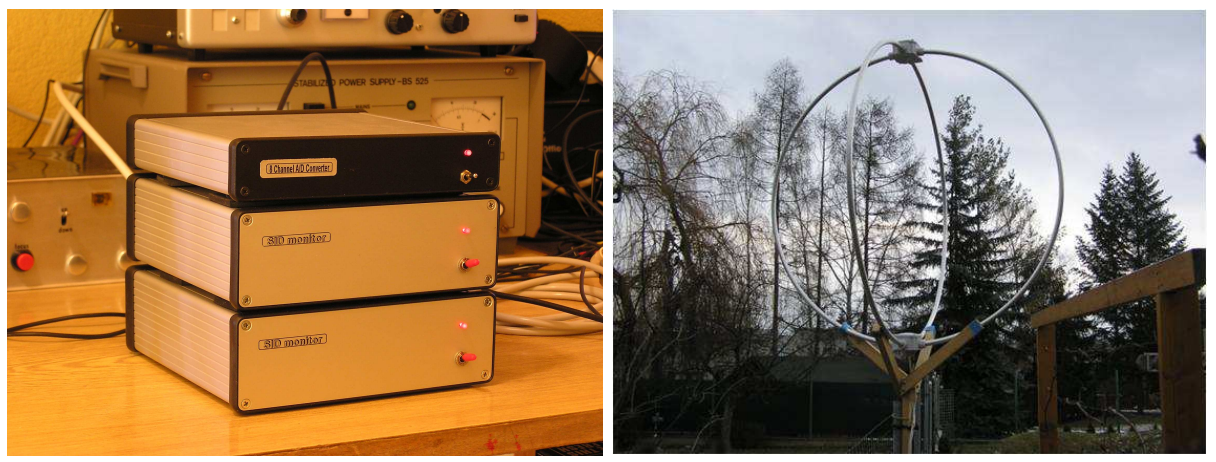

Figure 1: Instrumentation used for the indirect detection of GRB 060124A: the 2-channel receiver (left) and a loop antenna (right).

\section{Previous VLF detections of GRBs}

Previous reported VLF detections of GRBs were as follows. (i) SGR1806: detection of a Sudden Ionospheric Disturbance [1], (ii)GRB030329 observed as a Sudden Ionospheric Disturbance (SID) [2]. Although there were numerous efforts to detect GRBs by VLF and despite the fact that the necessary instrumentation is inexpensive, this field still remains little exploited.

\section{Physics behind ionospheric detection}

The solar particle stream, solar wind, shapes and controls the Earths magnetic envelope - the magnetosphere- and increases heat in the aurora zones. But not all ionospheric variability is caused by solar or geomagnetic disturbances. The ionosphere is not a constant 'mirror in the sky'. The E layer (100-200 km above ground) and the F1 layer (170-200 km) usually behave in regular, solarcontrolled way, but the F2 layer $(250-350 \mathrm{~km})$ does not. It is the F2 layer, which has the greatest density of free electrons, and is potentially the most effective reflector of radio waves ([3]).

The ionossperic D layer plays in the GRB detections an important role, as the detection of $\mathrm{X}$-ray and gamma-ray triggers is based on the measurement (monitoring) of reflected radio waves from this layer. The ionospheric D layer is not transparent for radio VLF waves (frequencies $3 \mathrm{kHz}$ to $30 \mathrm{kHz}$ ) and behaves like a mirror. If the transmitter is at large distance (800 to $2000 \mathrm{~km}$ ) then the radio waves are guided like in a waveguide consisting of the D layer and the earth surface. Any change in the quality of this waveguide results then in the signal change in the SID monitor. The change can be positive but in some cases such as the sudden phase anomaly also negative.

\section{Discussion}

The conditions to detect GRB with SID monitor in VLF can be briefly listed as follows. (i) The presence of the D layer of the ionosphere, (ii) The suitable combination of the GRB position (RA, DEC) and time and hence direction and angle of the incoming gamma-ray radiation in relation to the D layer, and (iii) The fluence and duration of the GRB. 


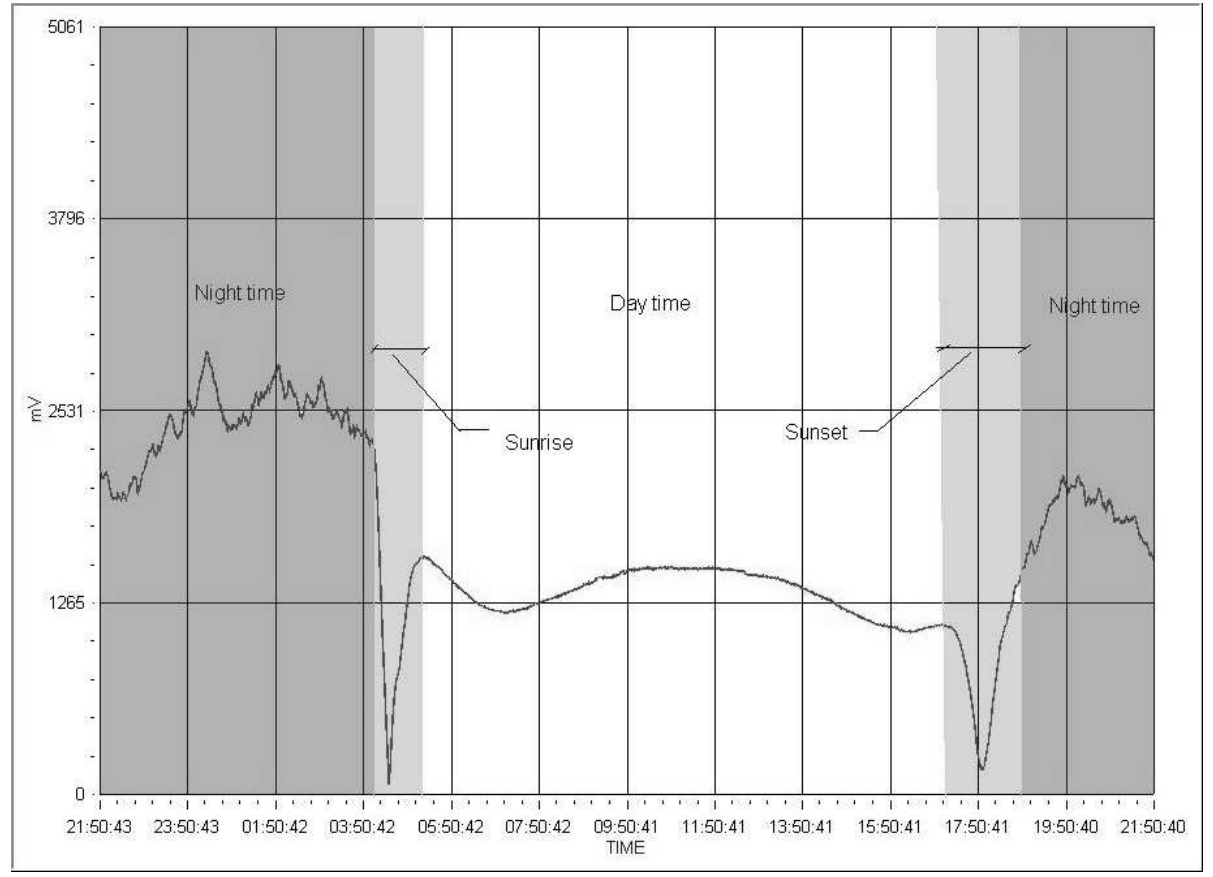

Figure 2: This picture shows the typical behaviour of the ionosphere during one day. Note the different behavior at night with absence of the D layer.

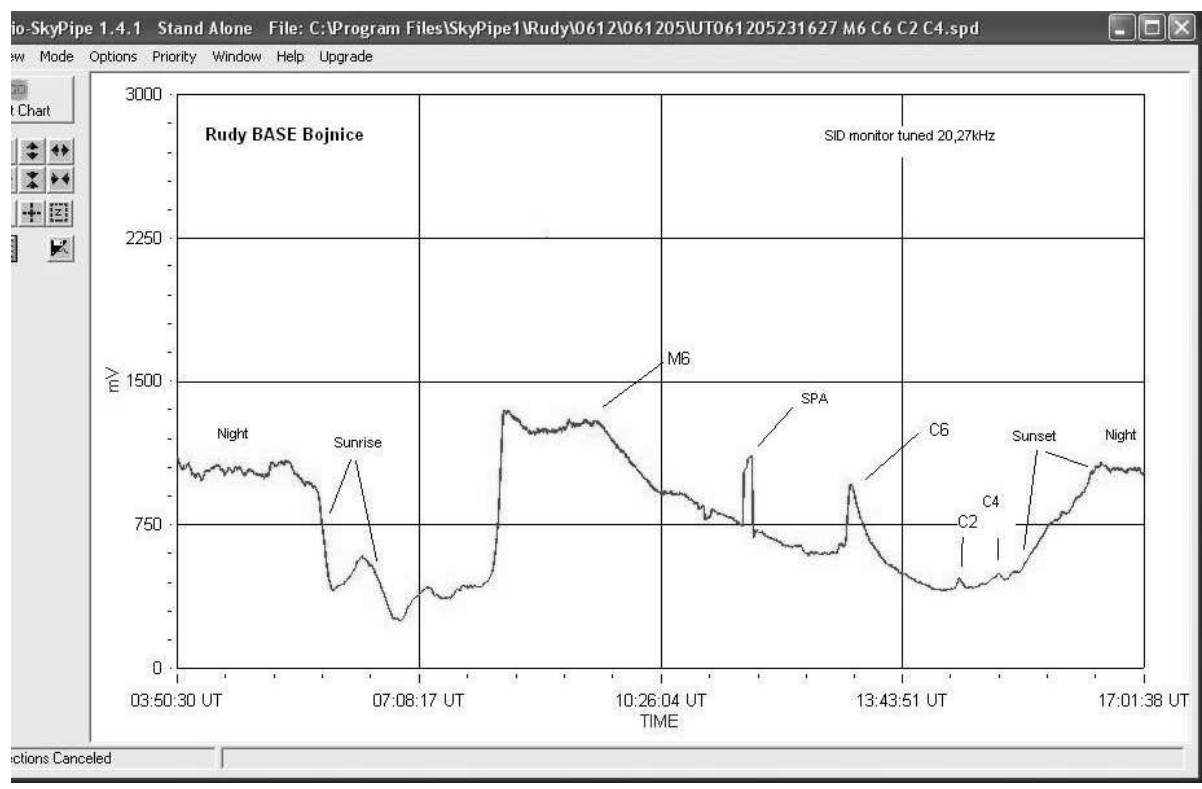

Figure 3: Demonstration of the possibility and sensitivity of the method. Four Solar Flares (SF) are visible with intensities M6, C6, C2, C4, exactly corresponding to the measurements by the GOES satellite. The peak related to the SF C4 occurs around 15:03UT, which is nearly the detection time of GRB060124A, on Jan 24, 2006, confirming that even at the time of the decay of the D ionospheric layer reliable measurements are feasible. 


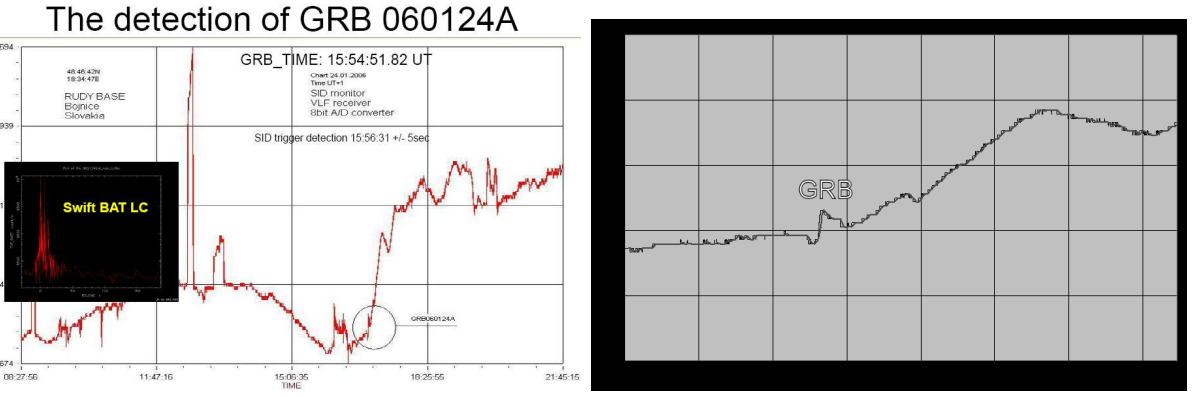

Figure 4: The detection of GRB 060124A

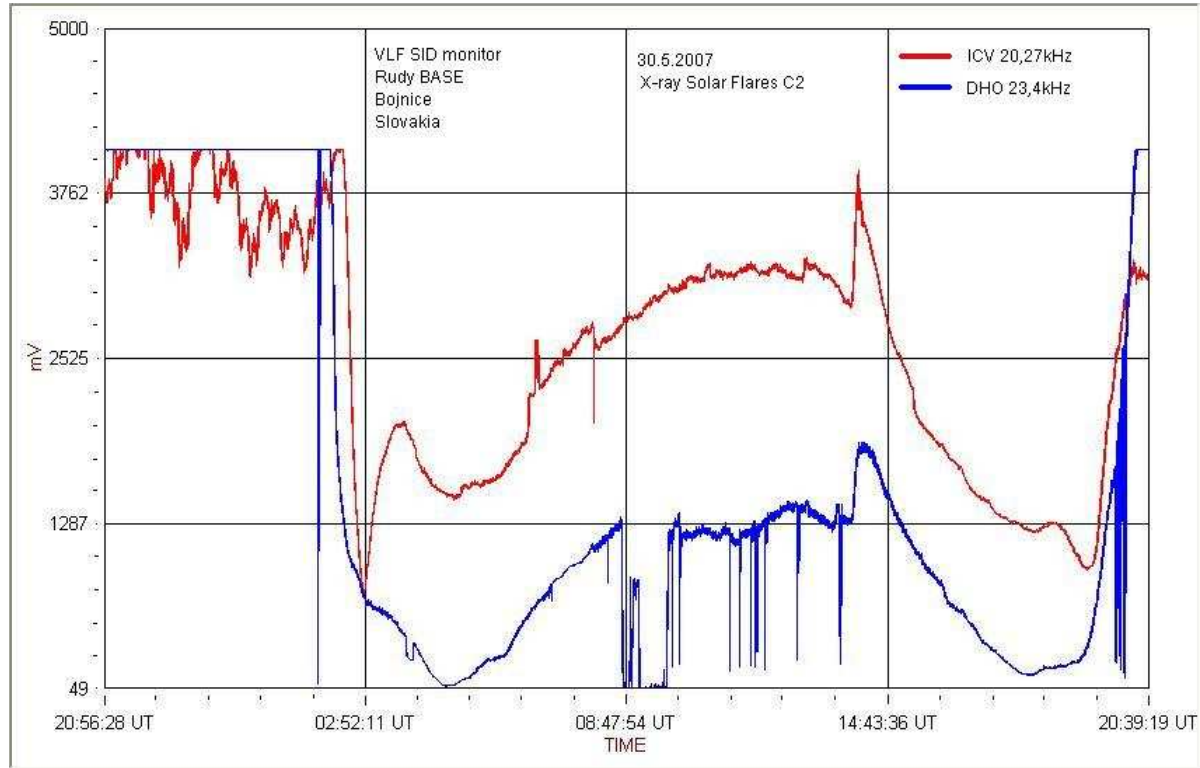

Figure 5: This picture shows (for comparison) the SID from C2-class solar flare (represented by the peak at around 14:40 UT) erupted from sunspot 958 .

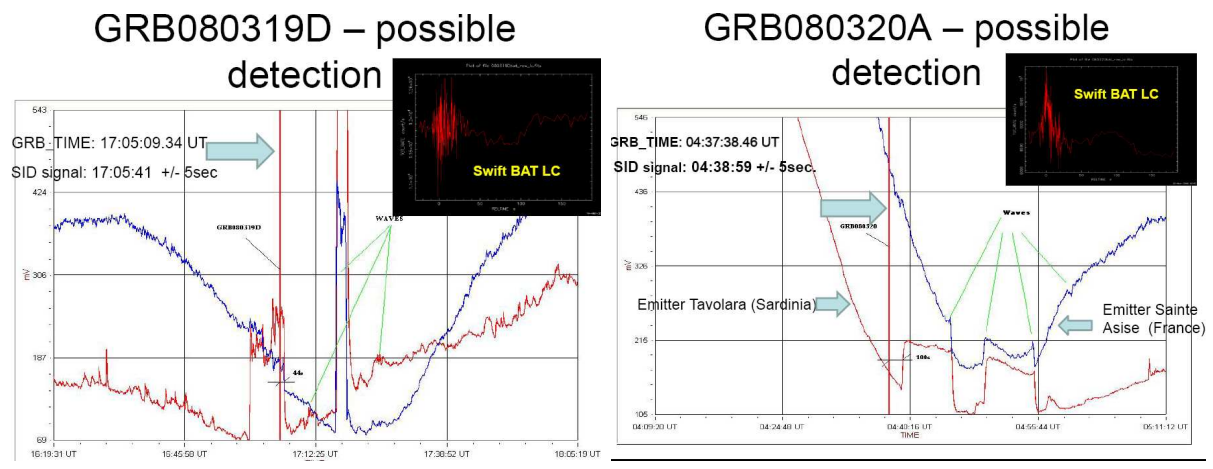

Figure 6: The probable detection of GRB080319D (left) and GRB080320A (right) with indicated probable propagating ionospheric waves caused by the GRB. 

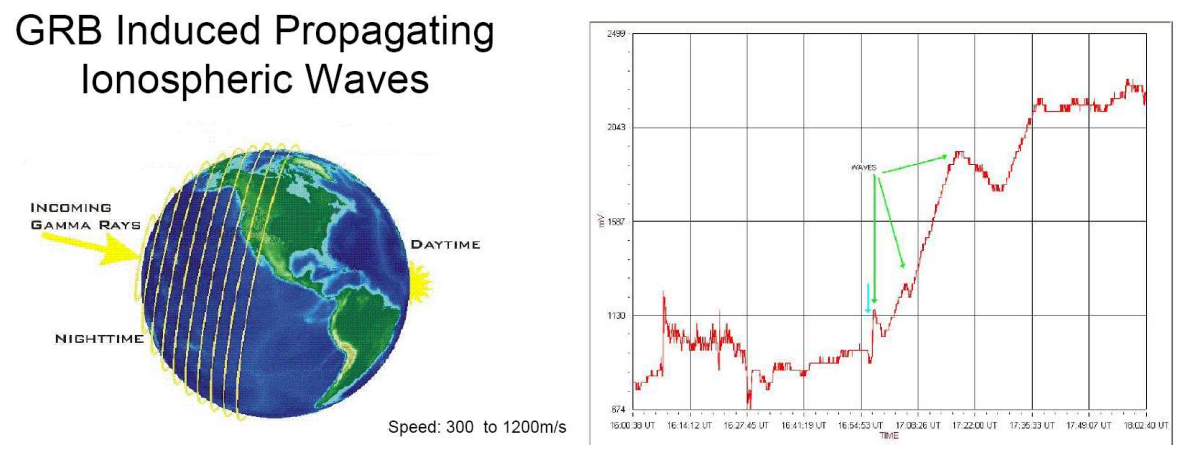

Figure 7: The principle of propagating ionospheric waves triggered by GRB (left) and the appearance of the propagating ionospheric waves for GRB060124A (right).

The detection statistics can be briefly discussed as follows. The recent detection rate of GRBs is about 130 in a year. For one observing station, the number of GRBs occuring during the presence of a D layer and in the field of view is about 20 This is ideal number, the real one is less than 10 due to occassional non-availability of transmitters and other technical and observational issues.

\section{Conclusions}

The independent and indirect detection of GRBs by their ionospheric response (SID, Sudden Ionospheric Disturbance) observed at VLF (Very Low Frequency) is feasible. We present and discuss examples of such VLF/SID detection in three cases: GRB 060124A, GRB080319D and GRB080320A. In addition, these measurements are in agreement with the scenarion of propagating ionospheric waves triggered by the relevant GRBs. Although few such detections have been already reported in the past, the capability of such alternative and indirect investigations of GRBs, as well as the possible contribution to analyses of GRBs still remains to be investigated in more details.

\section{Acknowledgments}

The GRB analyses described here are linked to the GRB analyses within the ESA PECS INTEGRAL Project 98023. Some parts are related to the grant of the Grant Agency of the Czech Republic 205/08/1207.

\section{References}

[1] P. Campbell et al., GCN, 2932, (2003).

[2] P. W. Schnoor et al., GCN, 2176, (2003).

[3] Rishbeth, H.; Müller-Wodarg, I. C. F.; Zou, L.; Fuller-Rowell, T. J.; Millward, G. H.; Moffett, R. J.; Idenden, D. W.; Aylward, A. D., Annales Geophysicae, 18, Issue 8, 945-956, (2000). 